Ernst P. Billeter

Grundlagen der erforschenden Statistik 


\title{
Grundlagen der erforschenden Statistik
}

Statistische Testtheorie

\author{
Ernst P. Billeter
}

Springer-Verlag

Wien · New York

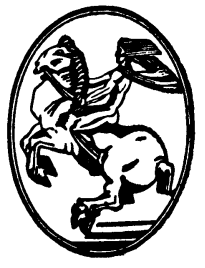




\section{Dr. ERNST P. BiLleter}

Ordentlicher Professor für Statistik, Operations Research

und Informatik an der Universität Freiburg/Schweiz

Direktor des Instituts für Automation und Operations Research

an der Universität Freiburg/Schweiz

Gastprofessor an der Pennsylvania State University, State College, Pa., U. S. A.

\section{Mit 13 Abbildungen}

Das Werk ist urheberrechtlich geschützt

Die dadurch begründeten Rechte, insbesondere die der Übersetzung, des Nachdruckes, der Entnahme von Abbildungen, der Funksendung, der Wiedergabe auf photomechanischem oder ähnlichem Wege und der Speicherung in Datenverarbeitungsanlagen, bleiben, auch bei nur auszugsweiser Verwertung, vorbehalten

(C) 1972 by Springer-Verlag/Wien

Softcover reprint of the hardcover 1st edition 1972

Library of Congress Catalog Card Number 73-185929 


\section{Vorwort}

Jede statistische Untersuchung bleibt Stückwerk, wenn sie nicht durch eine Wertung der erhaltenen statistischen Ergebnisse ergänzt wird. Aus diesem Grunde schließt sich das vorliegende Buch in natürlicher Weise den Ausführungen meiner beiden vorangegangenen Bücher über Grundlagen der Elementarstatistik und über Grundlagen der repräsentativen Statistik an.

Nach einer allgemeinen Einführung in die statistische Testtheorie werden einige der wichtigsten statistischen Tests in Theorie und Praxis dargestellt. Es wird dabei angestrebt, dem Statistiker die zur Wertung der Ergebnisse statistischer Untersuchungen notwendigen Grundlagen und Methoden an die Hand zu geben. Wiederum wurde, wie in meinen vorangegangenen Büchern, versucht, die Ableitungen nach Möglichkeit vollständig darzulegen, um dadurch das Verständnis auch des mathematisch weniger geschulten Lesers für die dargelegten Methoden zu erleichtern. Der behandelte Stoff beruht auf Vorlesungen für angehende Volks- und Betriebswirtschafter an der Universität Freiburg/Schweiz.

Ich möchte an dieser Stelle nicht versäumen, Herrn Dr. Wladimir VLACH, wissenschaftlicher Mitarbeiter am Institut für Automation und Operations Research der Universität Freiburg/Schweiz, für seine gewissenhafte und aufopfernde Arbeit bei der Drucklegung dieses Buches zu danken. Auch dem Verlag sei an dieser Stelle für seine Geduld und seine hervorragende Arbeit gedankt. Möge auch dieses Buch einem weiten Leserkreis die Welt der heute so wichtigen Statistik und ihrer Möglichkeiten eröffnen und damit das Interesse an statistischen Untersuchungen wecken.

Freiburg/Schweiz, Dezember 1971

Ernst P. Billeter 


\section{Inhaltsverzeichnis}

1. Wesen und Bedeutung der statistischen Testverfahren . . . . . . . . 1

1.1. Von der Stichprobentheorie zur Testtheorie .......... 1

1.2. Statistische Testtheorie und statistische Entscheidungstheorie . . . 2

1.3. Die Theorie von Neyman-Pearson . . . . . . . . . . . . 4

1.4. Entscheidungstheorie und Spieltheorie .......... 8

1.5. Vertrauensgrenzen . . . . . . . . . . . . . 10

1.6. Macht und Wirkungsgrad eines Tests . . . . . . . . . . 16

1.7. Kriterium der größten Mutmaßlichkeit ........... 18

1.8. Prüfplan-Kurve . . . . . . . . . . . . . . 21

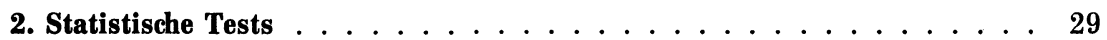

2.1. Allgemeines . . . . . . . . . . . . . . . 29

2.2. Verteilungsgebundene (parametrische) Tests ......... 35

2.2.1. "Student"-t-Test . . . . . . . . . . . 35

2.2.2. Fishers $F$-Test . . . . . . . . . . 52

2.2.3. Pearsons $\chi^{2}$-Test ................ 66

2.3. Verteilungsfreie (nicht-parametrische) Tests . . . . . . . . 79

2.3.1. Allgemeines . . . . . . . . . . . . 79

2.3.2. Allgemeine statistische Tests ............ 80

2.3.2.1. Verteilungs-Tests . . . . . . . . . 81

2.3.2.1.1. Rizzi-Test . . . . . . . . . 81

2.3.2.1.2. Kolmogorov-Smirnov-Test . . . . . 85

2.3.2.1.3. Smirnovs Test der größten Abweichung 93

2.3.2.1.4. Leerzellen-Test von David . . . . . . . 96

2.3.2.1.5. Wilcoxon-Test . . . . . . . . 100

2.3.2.1.6. Mann-Whitney-Test . . . . . . 106

2.3.2.1.7. Walsh-Test . . . . . . . . . 110

2.3.2.1.8. Kruskal-Wallis- $H$-Test . . . . . . . 113

2.3.2.1.9. Friedman-S-Test . . . . . . . 118

2.3.2.1.10. Terry-Hoeffding-Test . . . . . . . 123

2.3.2.1.11. Sequenz-Test . . . . . . . . 126

2.3.2.2. Korrelationstests . . . . . . . . . . . . 137

2.3.2.2.1. Hotelling-Pabst-Test . . . . . . . 137

2.3.2.2.2. Kendall-Test . . . . . . . . . . 139

2.3.2.2.3. Durbin-Watson-Test . . . . . . . 141 
Inhaltsverzeichnis

2.3.2.3. Trendtests . . . . . . . . . . . . 143

2.3.2.3.1. Daniels Test ... . . . . . . . 143

2.3.2.3.2. Mann-Kendall-Test . . . . . . . . 143

2.3.3. Binomiale Tests . . . . . . . . . . . . . . . 143

2.3.3.1. Cox-Stuart-Test . . . . . . . . . . 145

2.3.3.2. Noether-Test . . . . . . . . . . . 147

2.3.3.3. McNemar-Test . . . . . . . . . . . 150

2.3.3.4. Cochrans Q-Test .............. 153

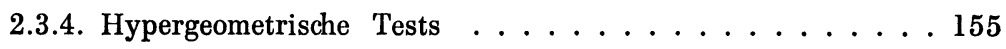

2.3.4.1. Fishers exakter Test . . . . . . . . 155

2.3.4.2. Westenberg-Mood-Test . . . . . . . . . 161

2.3.4.3. Blomqvists Test . . . . . . . . . . . 162

2.3.4.4. Wilks Leerzellen-Test . . . . . . . . . . 163

2.4. Transvariation ..................... 167

3. Testtheorie und Informationstheorie $\ldots \ldots \ldots 171$

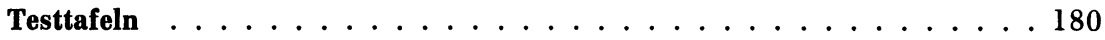

Tafel 1. Normalverteilung ................ 180

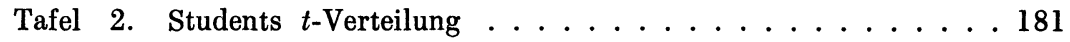

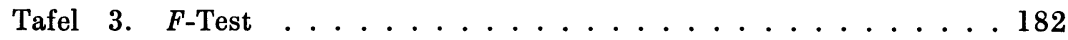

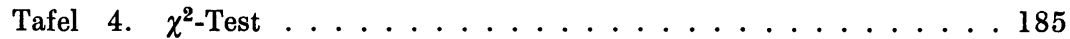

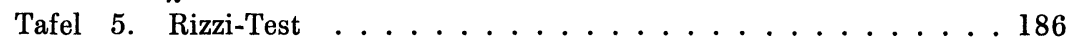

Tafel 6. Kolmogorov-Smirnov-Ein-Stichproben-Test . . . . . . . 187

Tafel 7. Smirnov-Test der größten Abweichungen .......... 188

Tafel 8. Davids Leerzellen-Test ............... 189

Tafel 9. Wilcoxon-Test, Ein-Stich-Proben-Fall . . . . . . . . 190

Tafel 10. Wilcoxon-Test, Zwei-Stichproben-Fall . . . . . . . . . 191

Tafel 11. Kumulierte Wahrscheinlichkeiten für den Wilcoxon-(Mann-

Whitney)-Zweistichproben-Rangfolge-Test . . . . . . . . 192

Tafel 12. Walsh-Test . . . . . . . . . . . . . . . 193

Tafel 13. Kruskal-Wallis-Tests . . . . . . . . . . . . . . . 194

Tafel 14. Friedman-Test .................. 195

Tafel 14 a. Friedmans $\chi^{2}$-Test . . . . . . . . . . . . . 196

Tafel 15. Terry-Hoeffding-Test I . . . . . . . . . . . 197

Tafel 15 a. Terry-Hoeffding-Test II . . . . . . . . . . . . . . . 198

Tafel 16. Kritische Werte von $U$ für Sequenz-Test . . . . . . . . . 199

Tafel 16 a. Sequenz-Test . . . . . . . . . . . . . . 200

Tafel 17. Hotelling-Pabst-Test ................ 201

Tafel 18. Kendalls Test ................... 202

Tafel 19. Durbin-Watson-Test . . . . . . . . . . . . . 203

Tafel 20. Zeichen-Test .................. 204

Tafel 21. Fishers exakter Test .............. 205

Literaturverzeichnis ... . . . . . . . . . . . 207

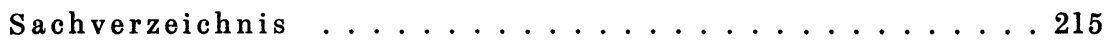

